\title{
Turbulent Poiseuille flow modeling by enhanced Prandtl-Driest mixing length
}

\author{
Bo-Hua Sun ${ }^{1}$ \\ ${ }^{1}$ School of Civil Engineering 8 Institute of Mechanics and Technology \\ Xian University of Architecture and Technology, Xian 710055, China \\ email: sunbohua@xauat.edu.cn
}

(Dated: Jan. 15, 2022)

\begin{abstract}
The turbulent Poiseuille flow between two parallel plates is one of the simplest possible physical situations, and it has been studied intensively. Different mixing lengths are used, but no one, to the best of our knowledge, has simultaneously considered the boundary conditions of two walls together with damping effects from both walls. In this paper, we propose an enhanced Prandtl-Driest mixing length that not only satisfies all of the boundary conditions, but it also includes both walls' damping effects as well. With our new formulations, we numerically solve the problem and, moreover, propose an explicit approximate solution. As applications of our solutions, the total velocity, total shear stress, energy dissipation density, energy balance, Kolmogorov scale law, and friction of turbulent Poiseuille flow are studied in detail. The study discovers that the high heels profiles of both mean and total velocity are universal for all wall-bounded turbulent flows.
\end{abstract}

Keywords: Turbulent flow, Poiseuille flow, Prandtl mixing length, high heels velocity profile, Reynolds number

\section{INTRODUCTION}

The theory of the turbulence boundary layer dates back 100 years. In 1921, Prandtl discovered that almost all of the boundary-layer movements in nature are turbulent rather than laminar [1-12]. The understanding of fully developed turbulence remains a major unsolved problem in physics. A challenge is how to use what we understand of this problem to build up a closure method; that is, to express the time-averaged turbulent stress tensor as a function of the time-averaged velocity field [1$3,13-32]$.

For the modeling of Reynolds stress, remarkable works include $[15,16]$, which shown that the Reynolds stress is a non local function in space of the time averaged velocity, involving an integral kernel, an extension of classical Boussinesq theory of turbulent viscosity. They do not use Prandtl mixing length but instead an equation for closure where the boundary condition is a consequence. Because of that they do not use a Prandtl equation, but instead an integral equation that reduces to a simpler one near the boundaries in a neighborhood defined precisely, a complete model where boundary conditions on the two walls are taken into account, however $[15,16]$ have not included the damping effects from both walls.

For the modeling of mean velocity profiles, notable works include [17, 18], and all build on the multi-layer concept of turbulent wall flows. For the Reynolds stresses (including Reynolds shear stress and normal stresses), [19] developed a similar multi-layer model for channels and pipes by using the symmetry argument. Recently, regarding the Reynolds-number scaling of near-wall fluctuations, [20] and [21] proposed the "law of bounded dissipation" and showed that near-wall peaks of turbulent dissipations, pressure intensities, fluctuation velocity intensities, etc. are all bounded for asymptotically high
Reynolds numbers, differing from the view of infinite logarithmic growth by [22] and references therein.

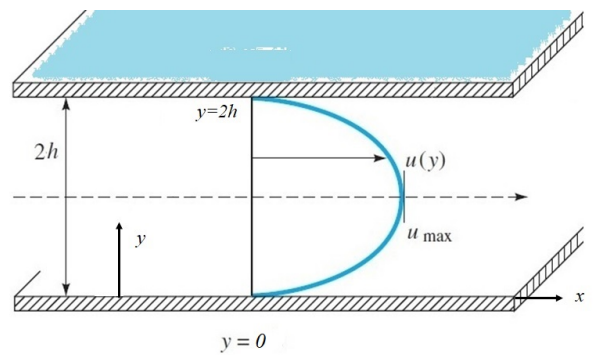

FIG. 1: Turbulent Poiseuille flow.

The turbulent Poiseuille flow between two parallel plates as shown in Fig.1 is one of the simplest possible physical situations, and it has been studied intensively $[1,2]$. Among those studies, some have adopted the Prandtl mixing length, such as Prandtl [9], who studied the same problem with the Prandtl mixing length by taking into account boundary conditions: $\ell_{\text {Prandtl }}(0)=0, \ell_{\text {Prandtl }}(2 h)=0$, and proposed mixing length as $\ell_{\text {Prandtl }}=\kappa y[1-y /(2 h)]^{6 / 7}$. Others used the Prandtl mixing length with a damping function, such as Absi, who investigated the turbulent Poiseuille flow with a van Driest mixing length [combining the Prandtl mixing length $(\ell=\kappa y)$ with a damping function $[1-\exp (-y / A)]]$, namely, $\ell_{A b s i}=\kappa y[1-\exp (-y / A)]$. However, this model is only valid for the bottom wall at $y=0$, but fails for the top wall at $y=2 h$.

Therefore, it is still a great challenge to propose a mixing-length model that can give an accurate prediction in the whole domain of wall coordinates $y$, which is the task of this study.

The rest of this paper is organized as follows. In Section 2, we propose an enhanced Prandtl-Driest mixing 
length. Section 3 completes formulations and we find integration solutions and an approximate analytical solution of mean motion. In Section 4, we calculate the turbulent viscosity, energy dissipation density, and Kolmogorov scaling law. Section 5 presents the velocity fluctuations and total velocity field. In Section 6, we investigate the Reynolds stress and total shear stress and, in Section 7, we construct the moment of velocity fluctuation and energy balance. In Section 8, we obtain the friction of the turbulent Poiseuille flow between two parallel plates. Finally, a discussion is presented and conclusions are drawn in the last two sections. As the essential part of this paper, a Maple code is provided.

\section{ENHANCED PRANDTL-DRIEST MIXING LENGTH}

Here, we consider a plane-parallel turbulent flow along an unbounded smooth plane surface (wall) as shown in Fig.1, and we take the direction of the flow as the $x$ axis and the plane of the surface as the $x z$ plane, so $y$ is the direction orthogonal to the surface. Assuming that the turbulent flow is steady with the pressure gradient along the $x$ axis, the $y$ and $z$ components of the mean velocity are zero, and all of the quantities depend only on $y$. The pressure gradient drives against the shear stresses at the two walls.

According to the Prandtl mixing-length theory [9], the Reynolds stress is proposed to be

$$
\tau_{x y}^{\prime}=-\rho \overline{u^{\prime} v^{\prime}}=\rho \ell^{2}\left|\frac{d \bar{u}}{d y}\right| \frac{d \bar{u}}{d y},
$$

where $\bar{u}$ is the mean velocity, $\mu$ the dynamical viscosity, $\rho$ the flow density, and $u^{\prime}$ and $v^{\prime}$ the velocity fluctuation components. The mixing length $\ell$ must have a length scale that should be of a form that satisfies the boundary condition $\overline{u^{\prime} v^{\prime}}=0$ at both bottom $(y=0)$ and top $(y=$ $2 h$ ) boundaries; hence, we can propose the mixing length as follows:

$$
\ell=\kappa y \varphi(y) \psi(y), \quad \text { and } \quad \varphi(2 h)=0,
$$

where $\kappa$ is a numerical constant, namely, the von Kármán constant, and $\varphi(y)$ is a dimensionless function that may take a different format. To satisfy the boundary condition, the function $\varphi$ can be proposed to be

$$
\varphi=\left(1-\frac{y}{2 h}\right)^{\gamma}=\left\{\begin{array}{cc}
0 & y=0 \\
0 & y=2 h
\end{array},\right.
$$

where the parameter $\gamma>0$, Prandtl [9] set $\gamma=6 / 7$; for simplicity, we set $\gamma=1$ in the present work.

Regarding the determination of function $\psi(y)$, we must take into account the fixed-wall damping effects. We consider an infinite flat plate undergoing simple harmonic oscillation parallel to the plate in an infinite fluid. According to Stokes [33], the amplitude of the motion diminishes with increasing distance from the surface (wall) as a consequence of the bottom-wall factor" $\psi_{B}=\exp \left(-\frac{y}{A}\right) "$ and top-wall factor " $\psi_{T}=\exp \left(-\frac{2 h-y}{A}\right)$ ", where $A$ is a constant depending the frequency of oscillation of the plate and kinematic viscosity $\nu$ of the fluid. Hence, in light of van Driest [27], we believe that when the plate is fixed and the fluid oscillates relative to the plate, the factor $[1-\exp (-y / A)]\left[1-\exp \left(-\frac{2 h-y}{A}\right)\right]$ must be applied to the fluid oscillation to obtain the damping effect on both bottom and top walls. Furthermore, van Driest pointed out that fully developed turbulent motion occurs only beyond a distance sufficiently remote from the wall, and eddies are not damped by the nearness of the wall. Indeed, near a wall, the damping factor is " $\psi=\psi_{B} \psi_{T}=[1-\exp (-y / A)]\left[1-\exp \left(-\frac{2 h-y}{A}\right)\right]$ " for each mean velocity fluctuation, and the Prandtl mixing length should be modified to the following,

$$
\ell=\kappa y \varphi \psi=\kappa y \varphi \psi_{B} \psi_{T}
$$

to take into account the mean motion all of the way to a smooth wall, where the bottom-wall van Driest damping function is

$$
\psi_{B}=\left[1-\exp \left(-\frac{y}{A}\right)\right]
$$

and the top-wall van Driest damping function is

$$
\psi_{T}=\left[1-\exp \left(-\frac{2 h-y}{A}\right)\right] .
$$

Introducing dimensionless parameters $\eta=y u_{\tau} / \nu, u^{+}=$ $\bar{u} / u_{\tau}, R_{\tau}=2 h u_{\tau} / \nu, A^{+}=A u_{\tau} / \nu$, and friction velocity $u_{\tau}=\sqrt{\tau_{w} / \rho}$, we have the dimensionless mixing length,

$$
\ell^{+}=\kappa \eta \varphi^{+} \psi^{+}
$$

where

$$
\begin{aligned}
\varphi^{+} & =1-\frac{\eta}{R_{\tau}} \\
\psi^{+}(\eta) & =\left[1-\exp \left(-\frac{\eta}{A^{+}}\right)\right]\left[1-\exp \left(-\frac{R_{\tau}-\eta}{A^{+}}\right)\right] .
\end{aligned}
$$

The $\ell^{+}$in Eq.7 is the mixing length to be used in this work, which is depicted in Fig.2.

\section{FORMULATIONS AND SOLUTIONS OF MEAN MOTION}

The Reynolds-averaged Navier-Stokes equations [4] of the turbulent Poiseuille flow under pressure gradient, $d p / d x$, are reduced to

$$
\begin{array}{r}
\rho \frac{d \overline{v^{\prime 2}}}{d y}+\frac{\partial p}{\partial y}=0 \\
\mu \frac{d^{2} \bar{u}}{d y^{2}}-\rho \frac{d \overline{u^{\prime} v^{\prime}}}{d y}-\frac{d p}{d x}=0
\end{array}
$$




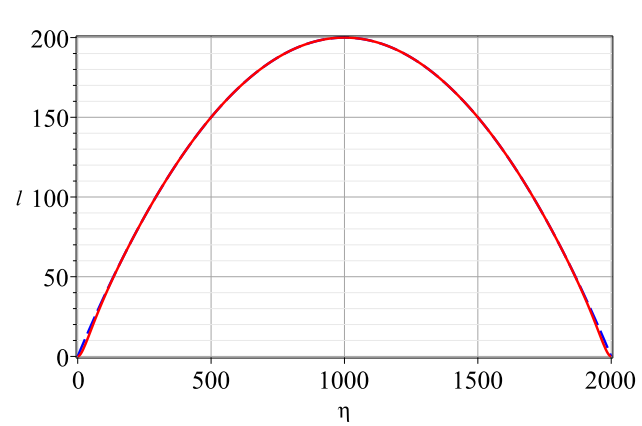

(a) Normal scale mixing length

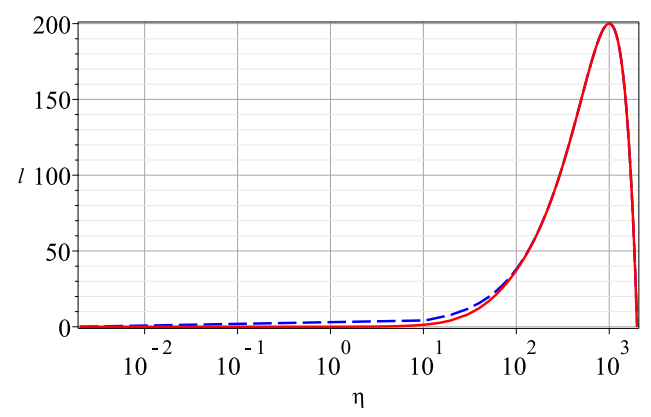

(b) Log-rescale mixing length

FIG. 2: Normal and log rescale of mixing-length profile. The solid line is from Eq.7 and the dashed line is for mixing length without van Driest damping functions, namely, $\kappa \eta\left(1-\eta / R_{\tau}\right)$.

and the boundary conditions, as shown in Fig.1 and 3, are

$$
y=0: \bar{u}=0, u^{\prime}=0, v^{\prime}=0, \text { and } \quad \mu \frac{d \bar{u}}{d y}=\tau_{w}
$$

and

$$
y=2 h: \bar{u}=0, u^{\prime}=0, v^{\prime}=0, \text { and } \quad \mu \frac{d \bar{u}}{d y}=-\tau_{w},
$$

where $\bar{u}$ is the mean velocity, $\mu$ the dynamical viscosity, $\rho$ the flow density, $p$ the pressure, and $u^{\prime}$ and $v^{\prime}$ the velocity fluctuation components. The pressure gradient must be negative, namely, $d p / d x<0$, to maintain the flow motion. $\tau_{w}$ is the wall friction force on a unit area of the surface. This force is clearly in the $x$ direction. The quantity $\tau_{w}$ is the constant flux of the $x$ component of momentum transmitted by the fluid to the surface per unit time.

Integration of Eq.10 yields

$$
\overline{v^{\prime 2}}+\frac{p}{\rho}=\frac{p_{0}}{\rho}
$$

where $p_{0}$ is a function of $\mathrm{x}$ only [1]. Because $\overline{v^{\prime 2}}$ is independent of $\mathrm{x}$ (by assumption), $\partial p / \partial x$ is equal to $d p_{0} / d x$. Both of these gradients should be independent of $x$ to avoid streamwise acceleration of the flow. Equation11 can be integrated to yield $\mu \frac{d \bar{u}}{d y}-\rho \overline{u^{\prime} v^{\prime}}=\frac{d p}{d x} y+C$. Applying the boundary condition in 13 , we have $\overline{u^{\prime} v^{\prime}}=0$,

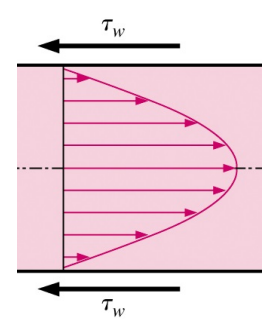

FIG. 3: Shear stress on the wall: $\tau_{x y}(y=0)=\tau_{w}$ and $\tau_{x y}(y=$ $2 h)=-\tau_{w}$.

leading to $C=\tau_{w}$. Hence, we have the following governing equation:

$$
\nu \frac{d \bar{u}}{d y}-\overline{u^{\prime} v^{\prime}}=\frac{1}{\rho} \frac{d p_{0}}{d x} y+\frac{\tau_{w}}{\rho},
$$

where $\nu=\mu / \rho$ is kinematic viscosity. Applying the boundary condition in 13 , we have the relation between the wall friction and pressure gradient:

$$
\frac{d p_{0}}{d x}=-\frac{\tau_{w}}{h}
$$

which indicates that the shear stress at the wall is determined by the pressure gradient and width of the channel only, which is one reason that this flow is less complicated than others [1]. Hence, Eq.15 is rewritten as

$$
\nu \frac{d \bar{u}}{d y}-\overline{u^{\prime} v^{\prime}}=\frac{\tau_{w}}{\rho}\left(1-\frac{y}{h}\right) .
$$

The first term on the left-hand side of Eq. 17 represents the effect of viscosity on the mean flow, whereas the second term is the Reynolds stress, namely, $\tau_{x y}^{\prime}=-\rho \overline{u^{\prime} v^{\prime}}$. In turbulent flow located some distance away from a wall, the Reynolds stress is of considerably greater magnitude than the viscous stress; however, the role of viscous stress increases as the distance to a smooth wall decreases, until, finally, at the wall, viscosity predominates.

Using the mixing length in Eq.2, Eq. 17 becomes $\nu \frac{d \bar{u}}{d y}+(\kappa y \varphi \psi)^{2}\left|\frac{d \bar{u}}{d y}\right| \frac{d \bar{u}}{d y}=\frac{\tau_{w}}{\rho}\left(1-\frac{y}{h}\right)$, which has two formats for different domains of $y$ and each of them has its own solution.

(a) In the domain $y \in[0, h]$, we have $\frac{d \bar{u}}{d y}>0$, and the governing equation is

$$
\nu \frac{d \bar{u}}{d y}+\ell^{2}\left(\frac{d \bar{u}}{d y}\right)^{2}=\frac{\tau_{w}}{\rho}\left(1-\frac{y}{h}\right) .
$$

(b) In the domain $y \in[h, 2 h]$, we have $\frac{d \bar{u}}{d y}<0$, and the governing equation is

$$
\nu \frac{d \bar{u}}{d y}-\ell^{2}\left(\frac{d \bar{u}}{d y}\right)^{2}=\frac{\tau_{w}}{\rho}\left(1-\frac{y}{h}\right) .
$$

So far, in the case of not including the damping functions, no complete solutions have been obtained for either Eq. 
18 or Eq. 19. Instead, asymptotic solutions have been constructed in two different regions (or sub-layers) $[1,2]$

In the inertial sub-layer, the first terms of Eq. 18 and Eq. 19 are neglected, leading to $\rho(\kappa y)^{2}\left(\frac{d \bar{u}}{d y}\right)^{2}=\tau_{w}$, the solution of which is a well-known Prandtl logarithmic law,

$$
\frac{\bar{u}}{u_{\tau}}=\frac{1}{\kappa} \log \left(\frac{y \bar{u}}{\nu}\right)+\alpha-\frac{1}{\kappa} \log \alpha,
$$

where $u_{\tau}=\sqrt{\tau_{w} / \rho}$ and $\nu=\mu / \rho$.

According to Nikuradse's famous experiments [? ], data fitting gives $\kappa=0.4$ and $\alpha=11.5$; hence, the Prandtl log-law is

$$
\frac{\bar{u}}{u_{\tau}}=5.75 \log \left(\frac{y \bar{u}}{\nu}\right)+5.5
$$

This expression becomes infinite at the boundary $y=0$ and is inapplicable at very small distances $y$ from the surface, since the effect of viscosity near the surface becomes non-negligible [3] .

To fix the singularity problem, traditionally a viscous sub-layer is introduced in which the viscosity of the fluid begins to be important. The second terms of Eqs. 18 and 19 can be neglected, leading to $\mu \frac{d \bar{u}}{d y}=\tau_{w}$, the solution of which is the Prandtl linear law: $\bar{u}=\tau_{w} y / \mu$.

Mathematically speaking, the above two segmental solutions, including linear and log-law solutions, are incomplete, because they are not whole domain solutions. Rather, it is a local solution of the matching area between the inertial sub-layer and viscous sub-layer of turbulence. Between the inertial region and viscous sub-layer, there is an intermediate region whose empirical solution has not been obtained $[2,3,23,24]$. We are going to fix it by complete integration method instead of the asymptotic solutions adopted in literature $[1-3,23]$.

With the dimensionless quantities, Eq.1 can be expressed as

$$
\frac{\tau_{x y}^{\prime}}{\rho u_{\tau}^{2}}=\left(\ell^{+}\right)^{2}\left|\frac{d u^{+}}{d \eta}\right| \frac{d u^{+}}{d \eta},
$$

and Eq. 18 can be rewritten as

$$
\begin{gathered}
\frac{d u^{+}}{d \eta}+\left(\ell^{+}\right)^{2}\left(\frac{d u^{+}}{d \eta}\right)^{2}=1-\frac{2 \eta}{R_{\tau}} \\
\eta \in\left[0, \frac{R_{\tau}}{2}\right]
\end{gathered}
$$

and the boundary condition

$$
\eta=0: u^{+}=0
$$

The solution of Eq.23 valid in the whole domain of $\eta$ has never been obtained, while only asymptotic solutions have been proposed [1, 2], which caused a buffer problem between segmental solutions.

From Eq.23, we can obtain

$$
\frac{d u^{+}}{d \eta}=\frac{2\left(1-\frac{2 \eta}{R_{\tau}}\right)}{1+\sqrt{1+4\left(\ell^{+}\right)^{2}\left(1-\frac{2 \eta}{R_{\tau}}\right)}} .
$$

Hence, the singularity-free solution is

$$
u^{+}=\int \frac{2\left(1-\frac{2 \eta}{R_{\tau}}\right)}{1+\sqrt{1+4\left(\ell^{+}\right)^{2} 2\left(1-\frac{2 \eta}{R_{\tau}}\right)}} d \eta
$$

Similarly, Eq. 19 can be rewritten as

$$
\begin{gathered}
\frac{d u^{+}}{d \eta}-\left(\ell^{+}\right)^{2}\left(\frac{d u^{+}}{d \eta}\right)^{2}=1-\frac{2 \eta}{R_{\tau}}, \\
\eta \in\left[\frac{R_{\tau}}{2}, R_{\tau}\right]
\end{gathered}
$$

and the boundary condition

$$
\eta=R_{\tau}: u^{+}=0
$$

From Eq.27, we can obtain

$$
\frac{d u^{+}}{d \eta}=\frac{2\left(1-\frac{2 \eta}{R_{\tau}}\right)}{1+\sqrt{1-4\left(\ell^{+}\right)^{2}\left(1-\frac{2 \eta}{R_{\tau}}\right)}} .
$$

Hence, the singularity-free solution is

$$
u^{+}=\int \frac{2\left(1-\frac{2 \eta}{R_{\tau}}\right)}{1+\sqrt{1-4\left(\ell^{+}\right)^{2}\left(1-\frac{2 \eta}{R_{\tau}}\right)}} d \eta .
$$

We can combine the derivatives in Eqs. (25) and (29) into a single form as follows:

$$
\begin{aligned}
\frac{d u^{+}}{d \eta} & =\frac{2\left(1-\frac{2 \eta}{R_{\tau}}\right)}{1+\sqrt{1-4\left(\ell^{+}\right)^{2}\left|\left(1-\frac{2 \eta}{R_{\tau}}\right)\right|}}, \\
\eta & \in\left[0, R_{\tau}\right]
\end{aligned}
$$

which is depicted in Fig. 4, showing that the velocity gradient is enormous near the wall, but decays rapidly to almost zero away from the wall. This is why the velocity profile near the wall must be obtained if we wish have a better understanding of the turbulence boundary layer.

We can also combine the integral solutions in Eqs.(26) and (30 into a single form as follows:

$$
u^{+}=\int \frac{2\left(1-\frac{2 \eta}{R_{\tau}}\right)}{1+\sqrt{1+4\left(\ell^{+}\right)^{2}\left|\left(1-\frac{2 \eta}{R_{\tau}}\right)\right|}} d \eta .
$$

To the best of our knowledge, the above integrations in the integral (32) cannot be completed exactly, but only 


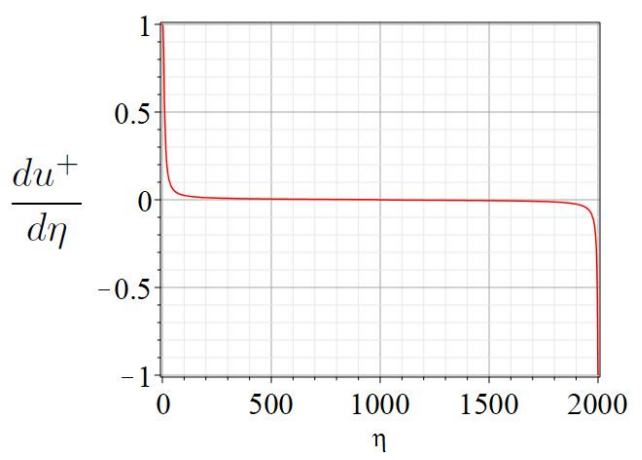

(a) Normal scale velocity gradient profile

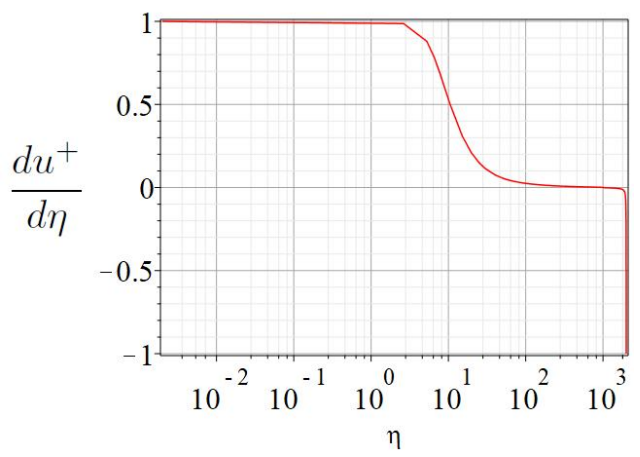

(b) Log-rescale velocity gradient profile

FIG. 4: Normal scale and log rescale of flow velocity gradient profile of turbulent Poiseuille flow.

approximately. One solution can be integrated by parts, yielding

$$
u^{+}=\sum_{n=1}^{\infty} \frac{(-1)^{n}}{n !} \eta^{n} \frac{d^{n} u^{+}}{d \eta^{n}}
$$

where $\frac{d u^{+}}{d \eta}=\frac{2\left(1-\frac{2 \eta}{R_{\tau}}\right)}{1+\sqrt{1-4\left(\ell^{+}\right)^{2}\left|\left(1-\frac{2 \eta}{R_{\tau}}\right)\right|}}$, and $\frac{d^{2} u^{+}}{d \eta^{2}}=$ $\frac{d}{d \eta}\left(\frac{d u^{+}}{d \eta}\right), . ., \frac{d^{n} u^{+}}{d \eta^{n}}=\frac{d}{d \eta}\left(\frac{d^{n-1} u^{+}}{d \eta^{n-1}}\right)$. This series solution can be computed to any order, but the series' convergence is poor and will need many terms to have a log law trend. We will not use this series solution in our computation.

Numerically, the integral (32) can be easily worked out, and a Maple code was written to compute the integral; the result is depicted in Fig. 5. This shows that the flow velocity rapidly increases away from the wall and decreases close to the center of the channel.

It is worth noting that the log-rescale mean velocity has a nice high heels profile because the boundary conditions of both walls are taken into account. One would not see the high heels profile if only one wall was considered. This high heels profile has never been seen in the literature before, to the best of our knowledge, and it is a universal feature of bounded flows.

Although the integral (32) cannot be completed exactly, we can try to obtain an approximate analytical solution. The author [31] obtained a closed-form solution

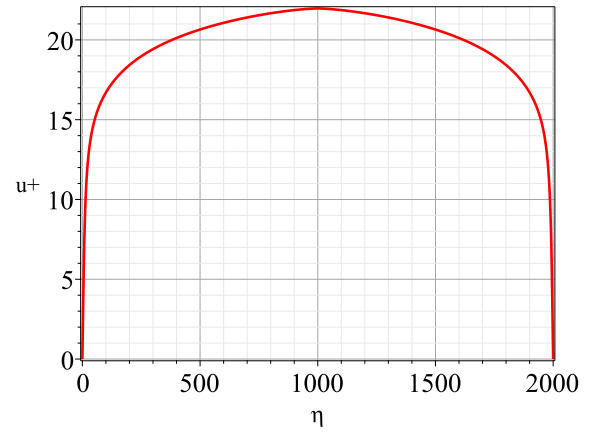

(a) Normal scale velocity profile

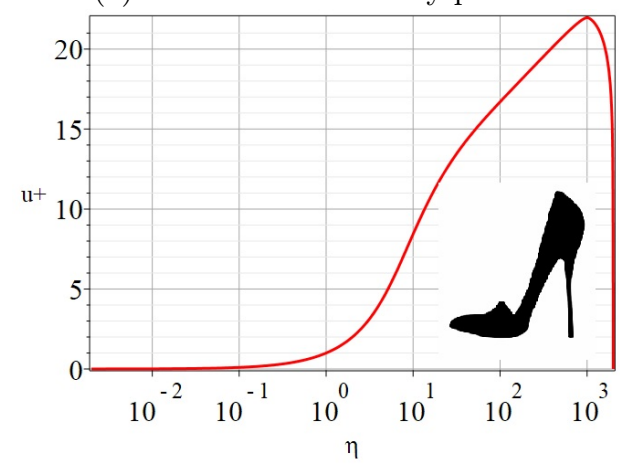

(b) Log-rescale velocity high heels profile

FIG. 5: Normal scale and log rescale of flow velocity profile of turbulent Poiseuille flow. The Maple code of the solution is $u^{+}(\eta)=\operatorname{int}\left(\frac{d u^{+}}{d \eta}\right.$, eta $=0 .$. a,numeric $)$.

for plane turbulent flow: $\frac{d u^{+}}{d \eta}+\kappa^{2} \eta^{2}\left(\frac{d u^{+}}{d \eta}\right)^{2}=1$, under boundary condition: $\eta=0: u^{+}=0$, its exact solution is given by: $u^{+}=\frac{1}{\kappa} \ln \left(2 \kappa \eta+\sqrt{1+4 \kappa^{2} \eta^{2}}\right)-\frac{2 \eta}{1+\sqrt{1+4 \kappa^{2} \eta^{2}}}$.

In light of the above exact solution, we can propose an approximate solution as follows:

$$
\begin{aligned}
u^{+} & \approx \frac{1}{\kappa} \ln \left[2 \ell_{C}^{+}+\sqrt{1+4\left(\ell_{C}^{+}\right)^{2}}\right] \\
& +\frac{\beta \ell_{C}^{+}}{1+\sqrt{1+4\left(\ell_{C}^{+}\right)^{2}}}, \quad \eta \in\left[0, R_{\tau}\right],
\end{aligned}
$$

where $(C, \beta)=(9,9)$, or $(C, \beta)=(26,8.8)$ and

$$
\begin{aligned}
\ell_{C}^{+} & =\kappa \eta \varphi^{+} \psi_{C}^{+} \\
\psi_{C}^{+} & =\left[1-\exp \left(-\frac{\eta}{C}\right)\right]\left[1-\exp \left(-\frac{R_{\tau}-\eta}{C}\right)\right] .
\end{aligned}
$$

This approximate analytical solution is valid in the whole domain $\eta \in\left[0, R_{\tau}\right]$, and it has not been seen in the literature to the best of our knowledge.

The solutions of Eqs. 26, 30, and 34 are depicted in Figs. 6 and its log rescale is the high heels velocity profile. It is remarkable to see that both the numerical integral solution and approximate analytical solution perfectly agree with both direct numerical simulation (DNS) solutions [24, 25] and experiments [26] in the domain 
of $\eta \in\left[0, R_{\tau} / 2\right]$. They do not have data in another half-domain $\left[R_{\tau} / 2, R_{\tau}\right]$, and hence, their curves are open rather than closed, as ours.

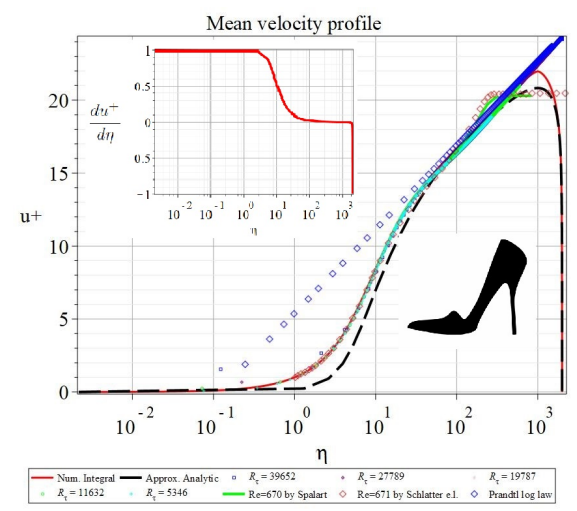

(a) Log-rescale mean velocity.

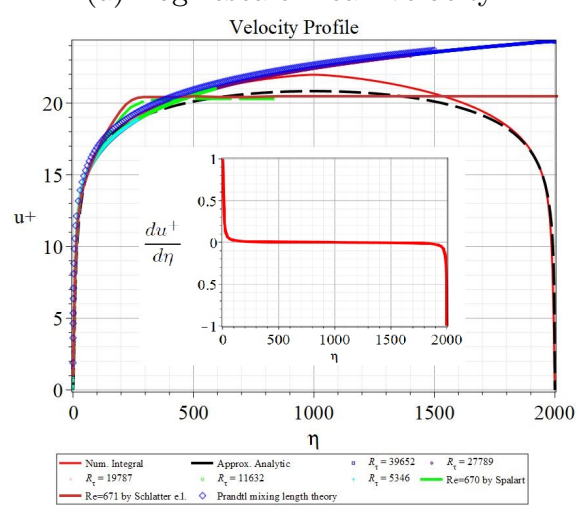

(b) Normal scale mean velocity.

FIG. 6: Log-rescale high heels velocity profile of turbulent Poiseuille flow and comparisons: numerical integration solution, approximate analytical solution, DNS solutions [24, 25], and experiments (all data with $R_{\tau}$ )[26].

\section{TURBULENT VISCOSITY, ENERGY DISSIPATION DENSITY, AND KOLMOGOROV SCALING LAW}

The turbulent viscosity is $\nu_{T}=\ell^{2}\left|\frac{d \bar{u}}{d y}\right|=\nu\left(\ell^{+}\right)^{2}\left|\frac{d u^{+}}{d \eta}\right|$, where $\frac{d u^{+}}{d \eta}=\frac{2\left(1-\frac{2 \eta}{R_{\tau}}\right)}{1+\sqrt{1+4 \kappa^{2} \eta^{2}\left(\varphi^{+} \psi^{+}\right)^{2}\left|\left(1-\frac{2 \eta}{R_{\tau}}\right)\right|}}$; hence,

$$
\nu_{T}=\frac{2 \nu \kappa^{2} \eta^{2}\left(1-\frac{\eta}{R_{\tau}}\right)^{2}\left|\left(1-\frac{2 \eta}{R_{\tau}}\right)\right|}{1+\sqrt{1+4 \kappa^{2} \eta^{2}\left(\varphi^{+} \psi^{+}\right)^{2}\left|\left(1-\frac{2 \eta}{R_{\tau}}\right)\right|}}
$$

This relation reveals that the turbulent viscosity is not constant, but rather changes with $\eta$. It is depicted in Fig. 7.

According to Landau [3], the mean energy flux density is $\langle q\rangle=\bar{u} \tau_{w}$, and the energy dissipation density in the

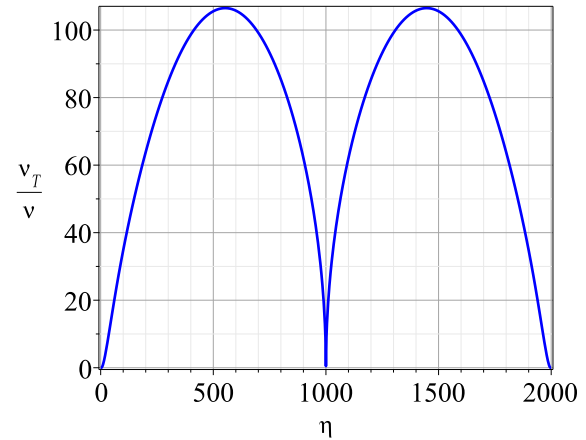

(a) Normal scale turbulent viscosity ratio

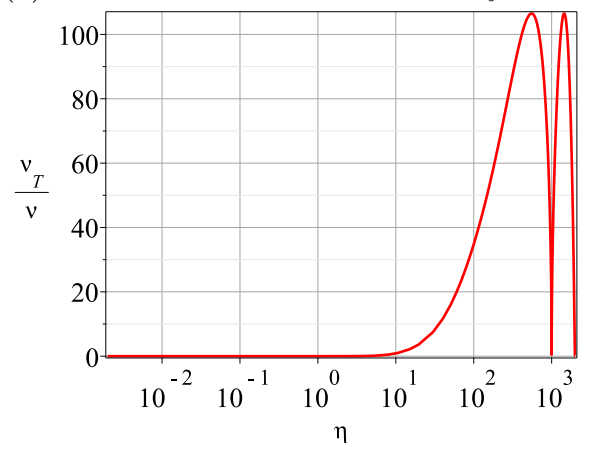

(b) Log-rescale turbulent viscosity ratio

FIG. 7: Turbulent viscosity ratio.

turbulent flow is

$$
\begin{aligned}
\varepsilon & =\frac{1}{\rho} \frac{d<q>}{d y}=\frac{1}{\nu}\left(\frac{\tau_{w}}{\rho}\right)^{2} \frac{d u^{+}}{d \eta}=\frac{u_{\tau}^{4}}{\nu} \frac{d u^{+}}{d \eta} \\
& =\frac{u_{\tau}^{4}}{\nu} \frac{2\left(1-\frac{2 \eta}{R_{\tau}}\right)}{1+\sqrt{1+4 \kappa^{2} \eta^{2}\left(\varphi^{+} \psi^{+}\right)^{2}\left|\left(1-\frac{2 \eta}{R_{\tau}}\right)\right|}}
\end{aligned}
$$

which gives $\lim _{\eta \rightarrow 0} \varepsilon=\frac{u_{\tau}^{4}}{\nu}$, i.e., a power law $u_{\tau}^{4}$. In contrast, Landau's energy dissipation density [3], $\varepsilon_{\text {Landau }}=$ $\left(\frac{\tau_{w}}{\rho}\right)^{3 / 2} \frac{1}{\kappa y}=\frac{u_{\tau}^{4}}{\nu} \frac{1}{\kappa \eta}$, is infinite at the surface $\eta=0$, indicating that maintaining turbulent flow requires supplying an infinite energy source, which is physically impossible. The energy dissipation density ratio $\epsilon=\frac{\varepsilon \nu}{u_{\tau}^{4}}$ is depicted in Fig.8.

According to Kolmogorov [29, 34-36], we have $E(k)=$ $1.5 \varepsilon^{2 / 3} k^{-5 / 3}$, namely,

$$
E(k)=\frac{1.5 \nu^{-2 / 3} k^{-5 / 3} u_{\tau}^{8 / 3}\left[2\left(1-\frac{\eta}{R_{\tau}}\right)\right]^{2 / 3}}{\left(1+\sqrt{1+4 \kappa^{2} \eta^{2}\left(\varphi^{+} \psi^{+}\right)^{2}\left|\left(1-\frac{2 \eta}{R_{\tau}}\right)\right|}\right)^{2 / 3}},
$$

where $E(k)$ is the kinetic energy per unit mass of fluid in eddies with wave number $k$. Hence, at the surface $\eta=0$, we have $E(k)_{\eta=0}=1.5 u_{\tau}^{8 / 3} k^{-5 / 3} \nu^{-2 / 3} . \quad \Sigma=$ $\frac{E}{1.5 \nu^{-2 / 3} k^{-5 / 3} u_{\tau}^{8 / 3}}$ is depicted in Fig.9, which shows that the turbulent energy dissipation is mainly concentrated 


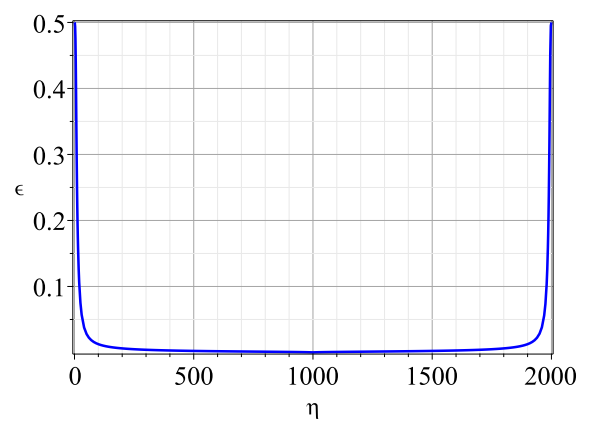

(a) Normal scale of energy dissipation density ratio

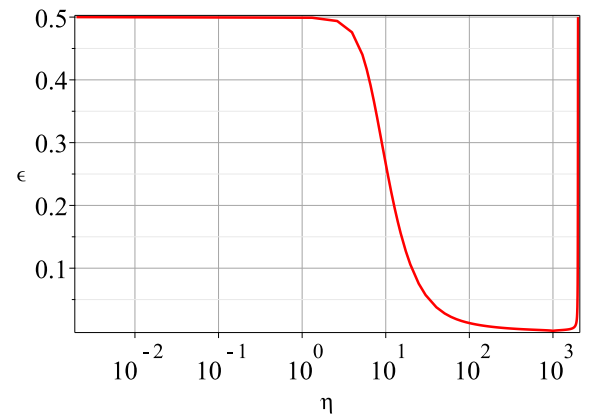

(b) Log rescale of energy dissipation density ratio

FIG. 8: Energy dissipation density ratio $\epsilon=\frac{\varepsilon \nu}{u_{\tau}^{4}}$ decreases to null rapidly as $\eta$ increases away from the surface.

in a narrow region close to the boundary surface and decays rapidly away from the surface.

\section{VELOCITY FLUCTUATIONS AND TOTAL VELOCITY FIELD}

In Prandtl mixing-length theory [2, 9], Prandtl assumes a greatly simplified model of the fluctuations, according to which the individual fluid elements are displaced in a mean distance (the mixing length) $\ell$ by the fluctuations, perpendicular to the main flow direction, but still retaining their momentum. The element that was initially at $y$, and is now at $y+\ell$, has a higher velocity than its new surroundings. The velocity difference is a measure of the fluctuation velocity in the $x$ direction:

$$
u^{\prime}=\bar{u}(y+\ell)-\bar{u}(y) \approx \ell \frac{d \bar{u}}{d y} .
$$

Prandtl assumed that the velocity fluctuation component $v^{\prime}$ is the same order of magnitude as $u^{\prime}$, namely, $v^{\prime} \sim u^{\prime}$, and therefore we have

$$
v^{\prime} \sim \frac{d \bar{u}}{d y},
$$

Using dimensionless quantities and $\frac{d u^{+}}{d \eta}=$ $\frac{2\left(1-\frac{2 \eta}{R_{\tau}}\right)}{1+\sqrt{1+4\left(\ell^{+}\right)^{2}\left|\left(1-\frac{2 \eta}{R_{\tau}}\right)\right|}}$, we have $u^{\prime+} \equiv u^{\prime} / u_{\tau}=\ell^{+} \frac{d \bar{u}^{+}}{d \eta}$ and

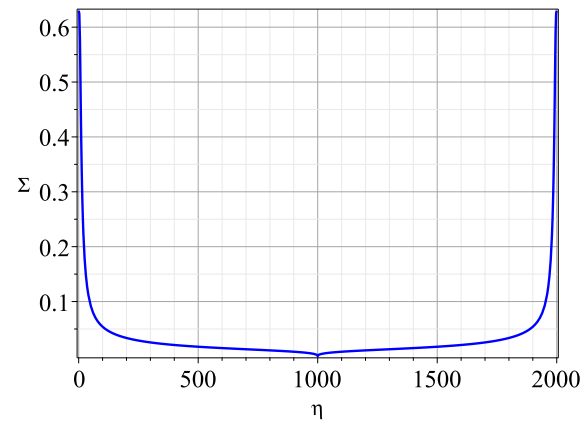

(a) Normal scale of kinetic energy ratio

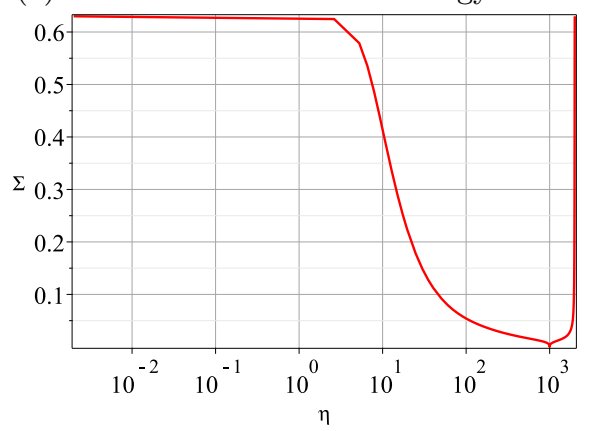

(b) Log rescale of kinetic energy ratio

FIG. 9: Kinetic energy ratio $\Sigma(\eta)=\frac{E}{1.5 \nu^{-2 / 3} k^{-5 / 3} u_{\tau}^{8 / 3}}$.

$v^{\prime+} \equiv v^{\prime} / u_{\tau}=\alpha \ell^{+} \frac{d \bar{u}^{+}}{d \eta}$, namely,

$$
\begin{aligned}
u^{\prime+} & =\frac{2 \ell^{+}\left|\left(1-\frac{2 \eta}{R_{\tau}}\right)\right|}{1+\sqrt{1+4\left(\ell^{+}\right)^{2}\left|\left(1-\frac{2 \eta}{R_{\tau}}\right)\right|}}, \\
v^{\prime+} & \sim \frac{\sqrt{2} \ell^{+}\left|\left(1-\frac{2 \eta}{R_{\tau}}\right)\right|}{1+\sqrt{1+4\left(\ell^{+}\right)^{2} \mid\left(1-\frac{2 \eta}{R_{\tau}}\right)}},
\end{aligned}
$$

where the symmetry of fluctuations about $\eta=R_{\tau} / 2$ has been applied. The fluctuations $u^{\prime+}$ or $v^{\prime+}$ are depicted in Fig.10.

We can verify that both velocity fluctuations satisfy the Reynolds-averaged conditions $\overline{u^{\prime+}}=0$ and $\overline{v^{\prime+}}=$ 0 . Since the problem is steady flow, now the Reynoldsaveraged conditions of quantity $\Phi$ are defined as follows:

$$
\overline{\Phi^{\prime+}}=\frac{1}{R_{\tau}} \int_{0}^{R_{\tau}} \Phi d \eta .
$$

Substituting Eqs.41 and 42 can prove this statement. Alternatively, it can be proved by numerical integration, e.g., by using the following Maple code: $\operatorname{int}\left(u^{\prime+}\right.$, $\eta=0 . . R_{\tau}$,nemeric), which yields null and can be interpreted easily because of the asymmetry of the velocity fluctuations about $y=R_{\tau} / 2$.

With the mean velocity and fluctuations, we have the total flow velocity components $U$ and $V$ in $x$ and $y$ directions, respectively:

$$
\begin{aligned}
& U^{+}=u^{+}+u^{\prime+}, \\
& V^{+}=v^{\prime+}
\end{aligned}
$$




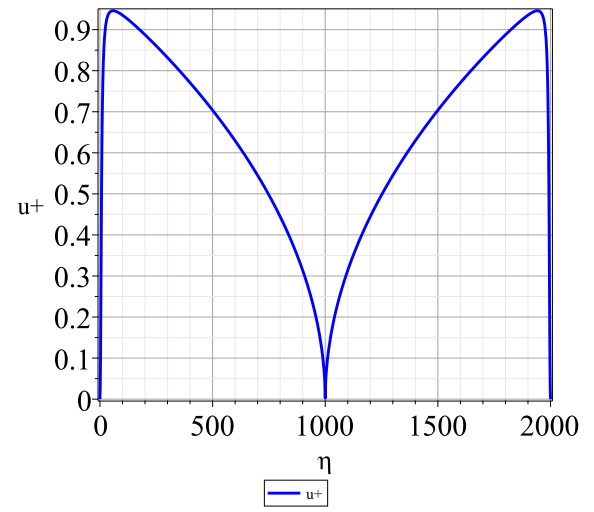

(a) Normal scale velocity fluctuations

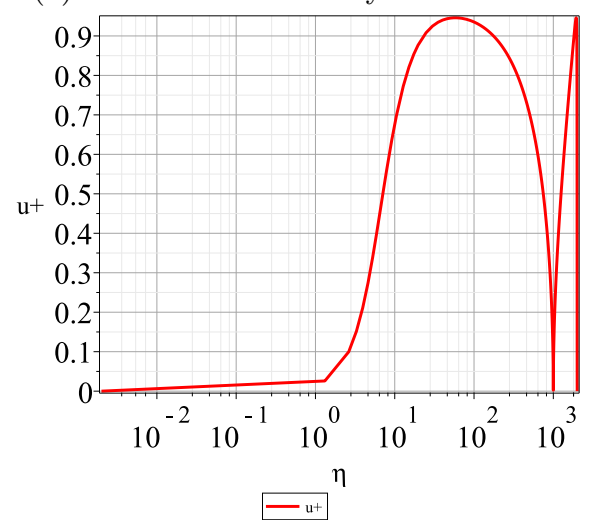

(b) Log-rescale velocity fluctuations

FIG. 10: Flow velocity fluctuations $u^{\prime+}$.

since $\bar{v}$ is assumed to be zero, where $U^{+} U / u_{\tau}$ and $V^{+}=$ $V / u_{\tau}$. The total flow velocity $U^{+}$and mean velocity $u^{+}$ are depicted in Fig.11.

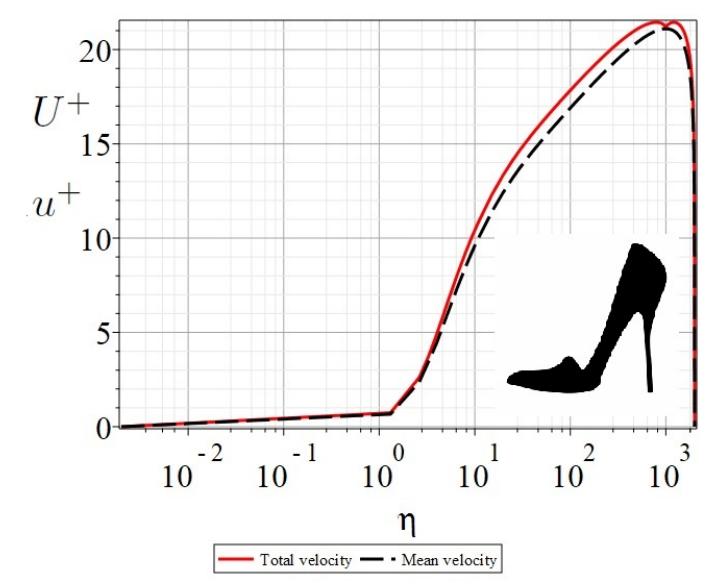

FIG. 11: Total flow velocity has a high heels profile and is slightly larger than the mean velocity due to the contribution of the fluctuations.

\section{REYNOLDS STRESS AND TOTAL SHEAR STRESS}

The Reynolds stress is given by $\tau_{x y}^{\prime}=$ $\rho u_{\tau}^{2} \kappa^{2} \eta^{2}\left(\varphi^{+} \psi^{+}\right)^{2}\left|\frac{d u^{+}}{d \eta}\right| \frac{d u^{+}}{d \eta}$. The Reynolds stress ratio $\frac{\tau_{x y}^{\prime}}{\rho u_{\tau}^{2}}$ is depicted in Fig. 12 .
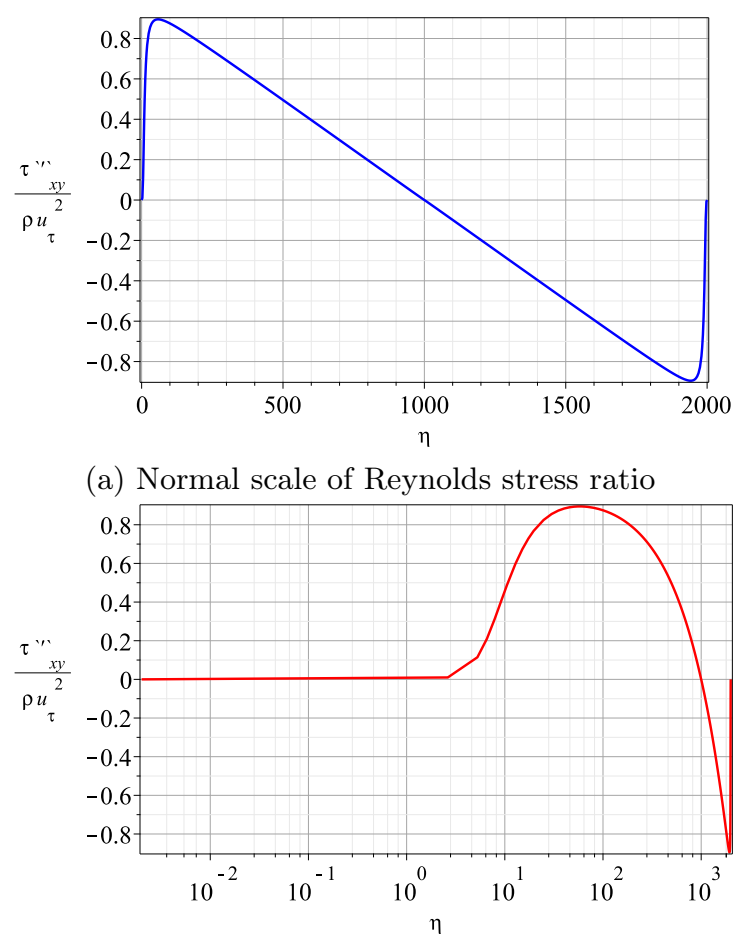

(b) Log rescale of Reynolds stress ratio

FIG. 12: Reynolds stress ratio $\frac{\tau_{x y}^{\prime}}{\rho u_{\tau}^{2}}$.

Total shear stress is given by $\tau_{x y}=\mu \frac{d \bar{u}}{d y}+\tau_{x y}^{\prime}$, namely,

$$
\tau_{x y}=\tau_{w}\left(1+\left(\ell^{+}\right)^{2}\left|\frac{d u^{+}}{d \eta}\right|\right) \frac{d u^{+}}{d \eta},
$$

where $\tau_{w}=\rho u_{\tau}^{2}$. The total shear stress is depicted in Fig. 13.

Figure13 reveals that the only difference in between are near the walls, while away from the walls they are almost equal.

\section{MOMENT OF VELOCITY FLUCTUATION AND ENERGY BALANCE}

The square of the streamwise flow velocity fluctuation with the wall distance, i.e., $\overline{\left(u^{\prime+}\right)^{2}}$, is given by

$$
\overline{\left(u^{\prime+}\right)^{2}}=\frac{4\left(\ell^{+}\right)^{2}\left(1-\frac{2 \eta}{R_{\tau}}\right)^{2}}{\left(1+\sqrt{1+4\left(\ell^{+}\right)^{2}\left|\left(1-\frac{2 \eta}{R_{\tau}}\right)\right|}\right)^{2}},
$$




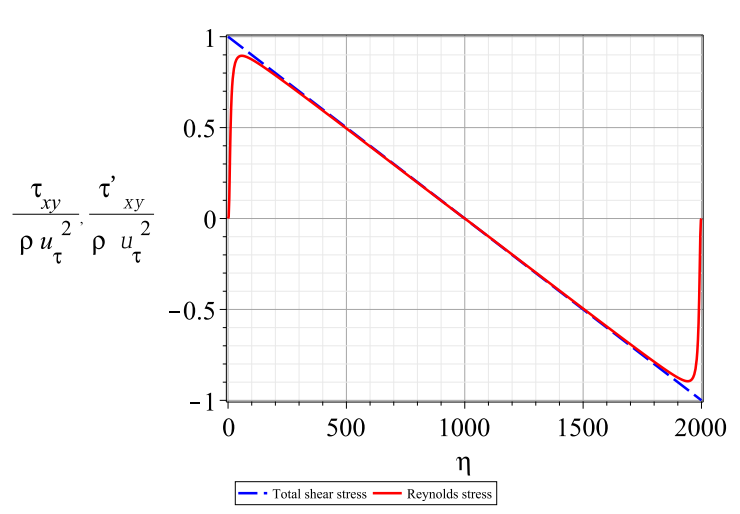

(a) Normal scale total shear stress

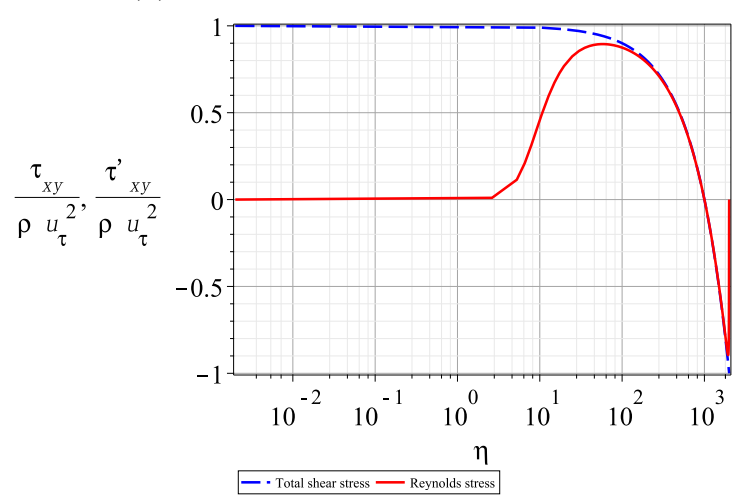

(b) Log-rescale total shear stress

FIG. 13: Total shear stress and Reynolds stress.

and $\overline{\left(v^{\prime+}\right)^{2}}$, as follows:

$$
\overline{\left(v^{\prime+}\right)^{2}} \sim \frac{2\left(\ell^{+}\right)^{2}\left(1-\frac{2 \eta}{R_{\tau}}\right)^{2}}{\left(1+\sqrt{1+4\left(\ell^{+}\right)^{2}\left|\left(1-\frac{2 \eta}{R_{\tau}}\right)\right|}\right)^{2}} .
$$

Both $\overline{\left(u^{\prime+}\right)^{2}}$ and $\overline{\left(v^{\prime+}\right)^{2}}$ have a profile similar to that shown in Fig.14, and their square roots, $\sqrt{\overline{\left(u^{\prime+}\right)^{2}}}$ and $\sqrt{\overline{\left(v^{\prime+}\right)^{2}}}$, are shown in Fig.15

The energy-balance equation can be be derived from the momentum-balance equation as follows:

$$
\underbrace{\left(1-\frac{2 \eta}{R_{\tau}}\right) \frac{d u+}{d \eta}}_{E_{e s}}=\underbrace{\left(\frac{d u+}{d \eta}\right)^{2}}_{E_{d d}}+\underbrace{\left(\ell^{+}\right)^{2}\left(\frac{d u+}{d \eta}\right)^{3}}_{E_{t p}},
$$

where $E_{e s}$ is the energy supply, $E_{d d}$ the direct dissipation, and $E_{t p}$ the turbulence production; all are depicted in Fig.16

The energy balance in Eq.16 reveals that the power due to the shear forces is divided into two parts. One is transformed directly to internal energy via viscous dissipation $\left(E_{d d}\right)$, while the second is used to generate turbulent fluctuation energy $\left(E_{t p}\right)$.

The turbulence production has a maximum of 0.2447 at $\eta=10.46$. At this distance from the wall, the direc$\mathrm{t}$ dissipation and turbulence production are equal. For

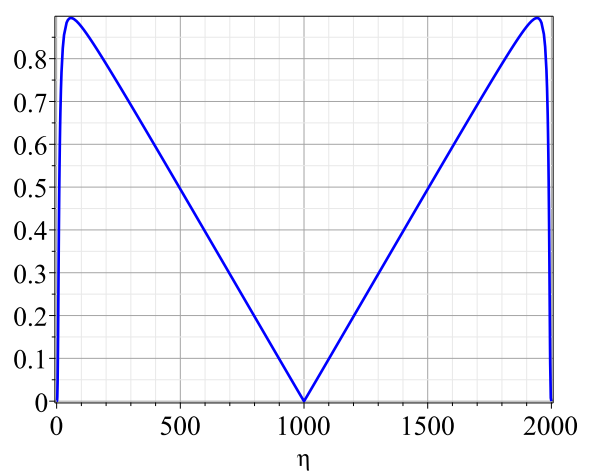

(a) Normal scale of $\overline{\left(u^{\prime+}\right)^{2}}$.

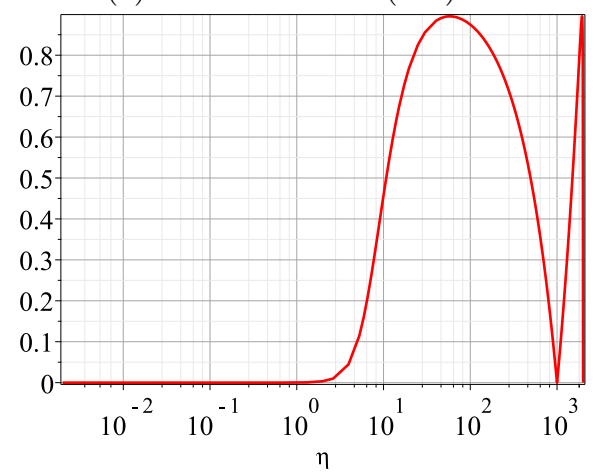

(b) $\log$ rescale of $\overline{\left(u^{\prime+}\right)^{2}}$.

FIG. 14: Square moment: $\overline{\left(u^{\prime+}\right)^{2}}$.

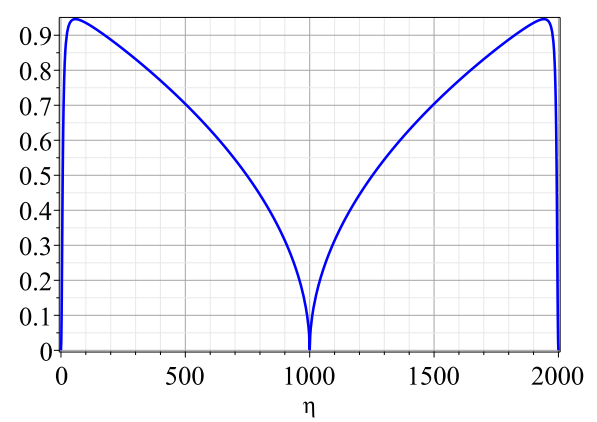

(a) Normal scale of $\sqrt{\overline{\left(u^{\prime+}\right)^{2}}}$.

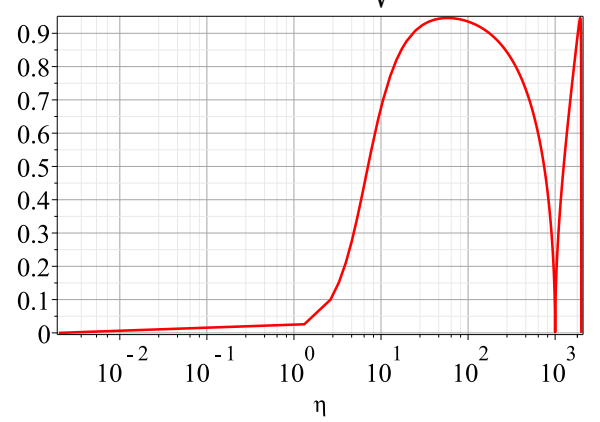

(b) Log rescale of $\sqrt{\overline{\left(u^{\prime+}\right)^{2}}}$.

FIG. 15: Square root: $\sqrt{\overline{\left(u^{\prime+}\right)^{2}}}$.

$\eta<10.46$, the direct dissipation dominates, and, for 


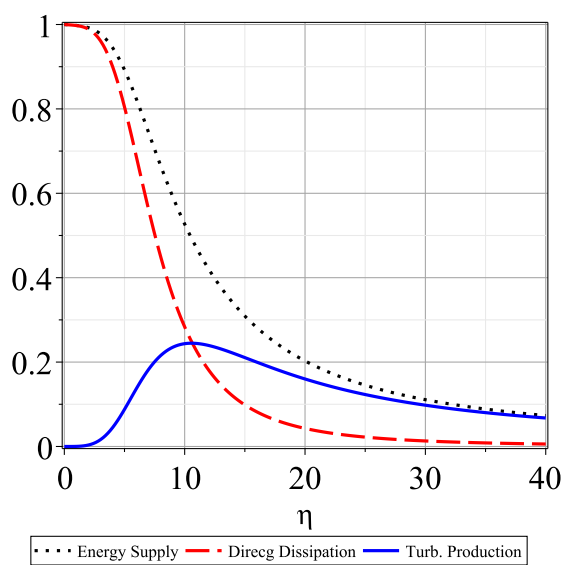

(a) Normal scale profile

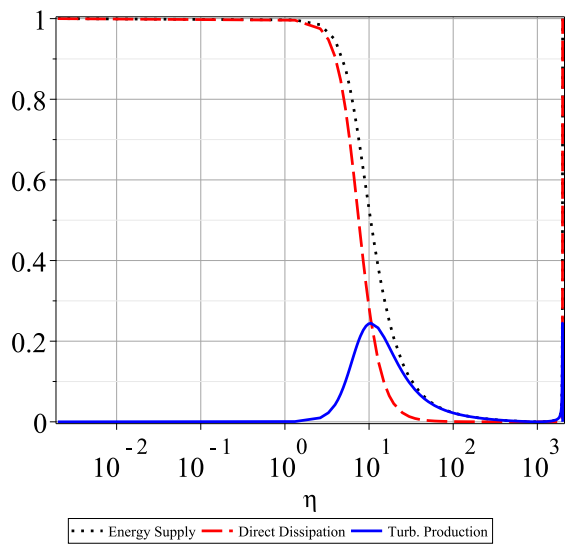

(b) Log-rescale profile

FIG. 16: Universal energy balance of mean motion.

$\eta>10.46$, the entire energy supply eventually provides the turbulence production as $\eta \rightarrow R_{\tau} / 2$, namely, closing the center of the channel [2]

The energy balance of the turbulent fluctuations can also be obtained and are shown in Fig.17.

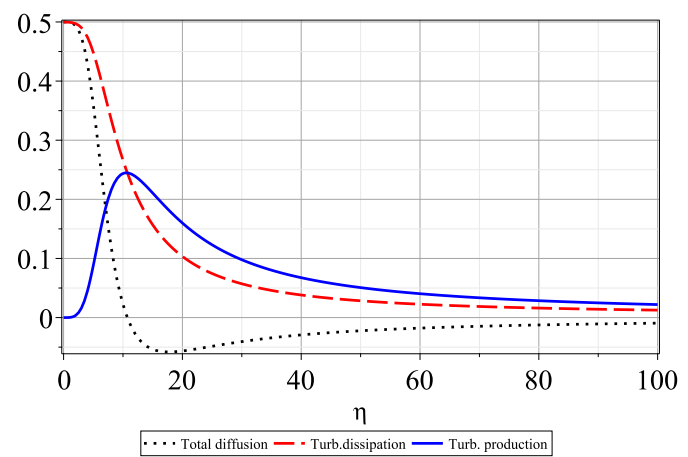

FIG. 17: Universal energy balance of turbulent fluctuations: Turbulence production + Total diffusion=Turbulent dissipation.
FRICTION OF TURBULENT POISEUILLE FLOW BETWEEN TWO PARALLEL PLATES

As an application of the proposed solutions, we now construct the dependence of the resistance coefficient, which is defined as $\lambda=\frac{2 h \Delta p / l}{(1 / 2) \rho \bar{u}^{2}}=2 h \frac{2 \rho u_{\tau}^{2}}{h} /\left(\rho \bar{u}^{2} / 2\right)=$ $8\left(u_{\tau} / \bar{u}\right)^{2}=8 /\left(u^{+}\right)^{2}$, which gives

$$
u^{+}=\sqrt{\frac{8}{\lambda}}
$$

The dimensionless Reynolds number is defined as $R e=$ $2 h \bar{u} / \nu=\left(2 h u_{\tau} / \nu\right) u^{+}$, and hence, $\left.\eta\right|_{y=2 h}=2 h u_{\tau} / \nu=$ $2 h\left(\bar{u} / u_{\tau}\right) / \nu=\operatorname{Re} /\left(2 u^{+}\right)$and $\left.2 \kappa \eta\right|_{y=2 h}=\kappa R e / u^{+}$. Applying Eq.50 to the above relation leads to

$$
\left.\eta_{h} \equiv \eta\right|_{y=2 h}=\frac{1}{2} R e \sqrt{\frac{\lambda}{8}} .
$$

The dependence of the resistance coefficient on the dimensionless Reynolds $R e$ number is given in implicit form by the equation

$$
\sqrt{\frac{8}{\lambda}}=\frac{1}{\kappa} \ln \left(2 \ell_{h}+\sqrt{\left(2 \ell_{h}\right)^{2}+1}\right)+\frac{\beta \ell_{h}}{1+\sqrt{\left(2 \ell_{h}\right)^{2}+1}},
$$

where

$$
\ell_{h}=\kappa \eta_{h}\left(1-\frac{\eta_{h}}{R_{\tau}}\right)\left[1-\exp \left(-\frac{\eta_{h}}{C}\right)\right]\left[1-\exp \left(-\frac{R_{\tau}-\eta_{h}}{C}\right] .\right.
$$

Equation 52 and the Prandtl $\log$-law, $1 / \sqrt{\lambda}=$ $2 \log (\operatorname{Re} \sqrt{\lambda})-0.8$, as well as others, are depicted in Fig. 18.

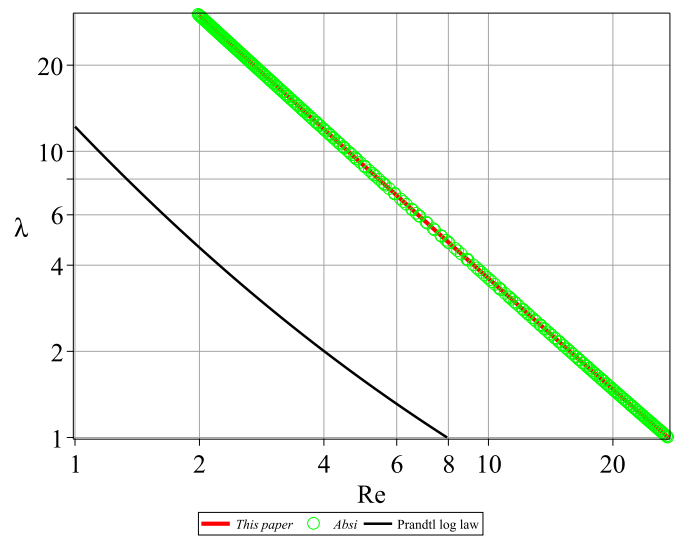

FIG. 18: Resistance coefficient of turbulent Poiseuille flow between two parallel plates.

\section{CONCLUSIONS}

To the best of the our knowledge, the enhanced mixing length is the first complete model that considers the 
boundary conditions of two walls and simultaneously includes the damping effects from both walls. The study shows that the high heels profiles of both mean and total velocitye are universal for all wall-bounded turbulent flows. The explicit approximate analytical solutions, total velocity, total shear stress, and friction of turbulent Poiseuille flow between two parallel plates has not be seen in the literature before. The investigation confirmed that the correct mixing length formulae must include all of the boundary conditions together with damping functions by multiplication. This study may help facilitate a better understanding of turbulence phenomena [28-32].

\section{ACKNOWLEDGMENTS}

This work was supported by Xi'an University of Architecture and Technology (Grant No. 002/2040221134). The author wishes to express his appreciation to Prof. X. Chen from Beihang University for providing some useful publications and to Mr. Zhe Liu for extracting the DNS and experimental data from Refs: [24-26], which are used to draw Fig. 6.

\section{Availability of data}

The data supporting the findings of this study are available from the corresponding author upon reasonable request.

\section{Interest Conflict}

The author declare that they have no conflict of interest.

[1] H. Tennekes and J.L. Lumley, A First Course of Turbulence, The MIT Press, Cambridge (1972).

[2] H. Schlichting, Boundary Layer Theory, fourth ed., McGraw-Hills, 1960 translated by J. Kestin.

[3] L.D. Landau and E. M. Lifshitz, Fluid Mechanics (2nd ed.) (Butterworth-Heinemann, 1987).

[4] O. Reynolds, On the dynamical theory of incompressible viscous fluids and the determination of the criterion. Philosophical Transactions of the Royal Society of London. 1895;186, 123-164. https://doi.org/10.1098/rsta.1895.0004

[5] L. Prandtl, On fluid motions with very small friction (in german). Third International Mathematical Congress, Heidelberg (1904)

[6] H. Blasius, Grenzschichten in Fljssigkeiten mit kleiner Reibung. Z. Math. Phys., 56(1908) 1-37.

[7] L. Prandtl, Bemerkungen über die entstehung der turbulenz, Z. Angew. Math. Mech. 1(1921):431-436.
[8] Th. von Kármán, Über leminere und turbulence Reibung (On Laminer and Turbulent Friction), Z. Angew. Math. Mech. 1 (1921)223.

[9] L. Prandtl, Bericht über Untersuchungen zur ausgebildeten Turbulenz, Z. Angew. Math. Mech. 5(2)(1925)136139.

[10] C. M. Millikan, A critical discussion of turbulent flows in channels and circular tubes, in Proceedings of the Fifth International Congress for Applied Mechanics, Harvard University and MIT, 1938 (Wiley, New York, 1939).

[11] J. Nikuradse, Untersuchungen über turbulente Strömungen in nicht kreisförmigen Rohren. Ing. Arch 1(1930) 306-332.

[12] J. Nikuradse, Gesetzmässigkeiten der turbulenten Stromung in glatten Rohren, Forschung auf dem Gebiet des Ingenieurwesens A (1934) 44.

[13] R. Baidya, J. Philip, N. Hutchins, J. P. Monty, and I. Marusic, Distance-from-the-wall scaling of turbulent motions in wall-bounded flows, Phy. Fluids 29(2017) 020712.

[14] R. Absi, A simple eddy visosity formulation for turbulent boundat payers near smooth walls, C.R. Mecanique, 337(2009)158-165.

[15] Y. Pomeau and M. Le Berre, Turbulence in a wedge: the case of the mixing layer, Phys. Rev. Fluids 6, 074603 (2021)

[16] Y. Pomeau and M. Le Berre, Turbulent plane Poiseille flow, EPJ, 2021.

[17] Z.S. She, X. Chen and F. Hussain, Quantifying wall turbulence via a symmetry approach: A Lie group theory, J. Fluid, Mech.827:322-356 (2017).

[18] B.J. Cantwell, A universal velocity profile for smooth wall pipe flow, J. Fluid Mech.,878:834-874(2019).

[19] X. Chen, X, F. Hussain and Z. S. She, Quantifying wall turbulence via a symmetry approach: Part II. Reynolds stresses, J. Fluid Mech. 850£ 401-438 (2018).

[20] X. Chen and SK.R. reenivasan, Reynolds number scaling of the peak turbulence intensity in wall flows, J. Fluid Mech., 908,R3 (2021)

[21] X. Chen and SK.R. reenivasan, Law of bounded dissipation and its consequences in turbulent wall flows, J. Fluid Mech., 933,A30(22)

[22] I. Marusic, W.J. Baars and N. Hutchins, Scaling of the streamwise turbulence intensity in the context of innerouter interactions in wall turbulence, Phys. Rev. Fluids, 2, 100502(2017)

[23] P. Luchini, Universality of the turbulent velocity profile, Phys.Rev.Lett. 118(2017) 224501.

[24] P. Schlatter, R. Örlü, Q. Li, G. Brethouwer, J.H.M. Fransson, A.V. Johnsson, P.H. Alfredsson and D.S. Henningson, Turbulent boundary layers up to $R e_{\theta}=2500$ studied through simulation and experiment. Phys. Fluids 21(2019) 051702.

[25] P. R. Spalart, Direct simulation of a turbulent boundary layer up to $R_{\theta}=1410$, J. Fluid Mech. 187 (1988)61-98.

[26] Willert, C., Soria, J., Stanilas, M., Klinner, J., Amili, O., Eisfelder, M., Cuvier, C., Bellani, G., Fiorini, T. \& Talamelli, A. 2017 Near-wall statistics of a turbulent pipe flow at shear Reynolds numbers up to 40 000. J. Fluid Mech. 826, R5.

[27] E.R. van Driest, On turbulent flow near a wall. J. Aeronaut. Sci. (Institute of the Aeronautical Sciences) 23(11)(1956) 1007-C1011 .

[28] B. H. Sun, The temporal scaling laws of compressible turbulence, Modern Physics Letters B, 30(23)(2016) 
1650297 (14 pages).

[29] B. H. Sun, Scaling laws of compressible turbulence, Appl. Math. Mech. -Engl. Ed., 38(6)(2017) 765-778.

[30] B. H. Sun, Thirty years of turbulence study in China. Applied Mathematics and Mechanics, 40(2)(2019) 193214.

[31] B. H. Sun, Revisiting the Reynolds-averaged NavierStokes equations, Open Physics 19 (2021) 853-862.

[32] B. H. Sun, Closed Form Solution of PlaneParallel Turbulent Flow Along an Unbounded Plane Surface. Preprints (2021) 2021110008 (doi: 10.20944/preprints202111.0008.v3); Fractals - Complex Geometry, Patterns, and Scaling in Nature and Society (accepted).

[33] G. G. Stokes, On the effect of the internal friction of fluids on the motion of pendulums, Trans. Cambridge Philos. Soc., 9 (1851).
[34] A. N. Kolmogorov, The local structure of turbulence in incompressible viscous fluid for very large Reynolds number. Dokl. Akad. Nauk SSSR, 30(1941), 299-303 (reprinted in Proc.R.Soc.Lond. A, 434(1991)9-13.)

[35] A. N. Kolmogorov, On degeneration (decay) of isotropic turbulence in an incompressible visous liquid. Dokl. Akad. Nauk SSSR, 31(1941) 538-540.

[36] A. N. Kolmogorov, Dissipation of energy in locally isotropic turbulence. Dokl.Akad. Nauk SSSR., 32(1941) 16-18 .(reprinted in Proc.R.Soc.Lond. A, 434(1991)15$17)$.

[37] K. Gersten and H. Herwig, Stömungsmechanik Grundlagen der Impuls-, Wärme-und Stoffübertragung aus asymptotischer Sicht. Vieweg-Verlag, Braunschweig, Wiesbaden (1992). 